\title{
Static and dynamical inhomogeneity at liquid - liquid phase transition of Se-Te mixtures
}

\author{
Y. Kajihara ${ }^{1}$, M. Inui ${ }^{1}$, K. Matsuda ${ }^{2}$, D. Ishikawa ${ }^{3}$, S. Tsutsui ${ }^{3}$, and A.Q.R. Baron ${ }^{3,4}$ \\ ${ }^{1}$ Graduate School of Integrated Arts and Sciences, Hiroshima University, 1-7-1 Kagamiyama, Higashi-Hiroshima 739-8521, Japan \\ ${ }^{2}$ Department of Physics, Graduate School of Sciences, Kyoto University, Sakyo-ku, Kyoto 606-8502, Japan \\ ${ }^{3}$ SPring-8 / JASRI, 1-1-1 Kouto, Sayo-cho, Sayo-gun, Hyogo 679-5198, Japan \\ ${ }^{4}$ SPring-8 / RIKEN, 1-1-1 Kouto, Sayo-cho, Sayo-gun, Hyogo 679-5198, Japan
}

\begin{abstract}
We have carried out $\mathrm{x}$-ray transmission and small-angle $\mathrm{x}$-ray scattering (SAXS) measurements of liquid Se70-Te30 mixture up to $1000{ }^{\circ} \mathrm{C}$ and $100 \mathrm{MPa}$ and inelastic $\mathrm{x}$-ray scattering (IXS) measurement of liquid Se50-Te50 mixture at SPring-8 in Japan. In this paper, we report the preliminary results. In liquid Se70Te30 at $6 \mathrm{MPa}$, with increasing temperature from $400{ }^{\circ} \mathrm{C}$, the density first normaly decreases but anomalously increases from $650{ }^{\circ} \mathrm{C}$. This anomalous density behaviour can be interpreted that the sample exhibits continuous transition from low-density phase to high-density one in this temperature region. As a proof of that, the zero-wavenumber-limit of SAXS intensity I(0) increases and shows maximum in this region, which means that the static density inhomogeneity arises due to phase transition. When the pressure is elevated, the density and I(0) curves shift to lower temperature side. The velocity of acoustic mode in Se50-Te50 estimated by IXS data is much higher than the ultrasonic sound velocity (so-called "fast sound" state) and the temperature dependences of the two velocities are totally different. But the ratio of the two velocities, the strength of "fast sound", seems to increase with approaching to the transition region and thus it seems to be a good sign of dynamical inhomogeneity.
\end{abstract}

\section{Introduction}

Liquid Se-Te mixtures are known to exhibit gradual change from 2-fold coordinated Se-like structure to 3fold coordinated Te-like one when the composition and/or the temperature of sample change ${ }^{1,2}$. This structural change accompanies anomalous thermodynamic features: density maximum and minimum $^{3}$, sound velocity minimum ${ }^{4,5}$ and heat capacity maximum $^{6}$. It also accompanies gradual change of the electronic properties ${ }^{7,8}$ and liquid Se-Te mixtures exhibit continuous semiconductor-metal (SM-M) transition. The phase diagram of Se-Te mixtures is shown in Fig.1. The SM-M transition (threshold conductivity is around $100 / \Omega$ $\mathrm{cm}$ ) is located in the middle of density maximum and minimum. These properties are also observed in the highpressure region ${ }^{9,10,11}$. In Se50Te50, as the pressure increases, the transition temperature gradually decreases from about $650{ }^{\circ} \mathrm{C}$ at ambient pressure to about $450{ }^{\circ} \mathrm{C}$ at $500 \mathrm{MPa}^{10}$.

To explain thermodynamic anomaly in Se-Te mixtures, existence of large fluctuations ${ }^{4}$ (inhomogeneity ${ }^{5}$ ) in the transition region are probable, but there have been no clear direct experimental observations of such density

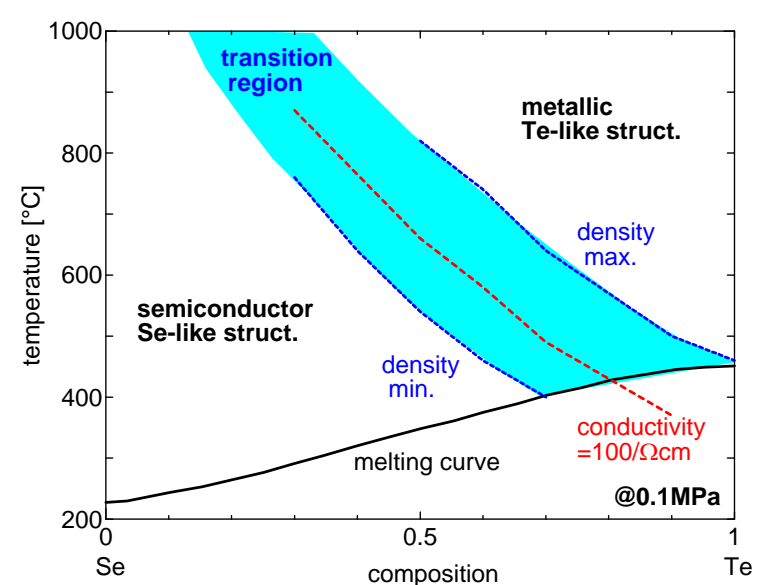

Fig. 1. Phase diagram of Se-Te mixure at ambient pressure. Solid line is a melting curve. Dotted lines are the points where the temperature dependence of the density shows minimum/maximum and dashed line is a point where the conductivity is $100 / \Omega \mathrm{cm}$. Hatched region is a continuous phase transition region. 
fluctuations for long time. Recently, we have succeeded to detect this density inhomogeneity in liquid Se50-Te50 mixture by SAXS measurement at SPring- $8^{12}$.

In this study, we have carried out SAXS measurement of Se70-Te30 mixture to support the previous results and IXS measurement of Se50-Te50 mixture to investigate the effect of this inhomogeneity in dynamical nature.

\section{Experiment}

The transmission and SAXS experiments of Se70-Te30 were carried out at the High Energy X-ray Diffraction beamline BL04B2 of SPring-8 in Japan. The scattered $\mathrm{X}$ ray was detected with an imaging plate located at $2.96 \mathrm{~m}$ distance from the sample position and accumulated for 20 min. The intensity of transmitted x-ray was detected with an ionization chamber. Se-Te samples were contained in a poly-crystalline sapphire cell, which was located in a high-pressure vessel equipped with single-crystalline diamond windows. The energy of incident x-ray was 61.7 $\mathrm{keV}$ and the sample thickness was $700 \mu \mathrm{m}$. The vessel was pressurized by He gas and the sample was heated by tungsten heaters.

The IXS measurement Se50-Te50 was carried out at the Inelastic Scattering beamline BL35XU of SPring-8. The sample was contained in a single-crystalline sapphire cell. Newly constructed high-pressure vessel equipped with a large Be window was used for this experiment. The energy of incident $\mathrm{x}$-ray was $21.75 \mathrm{keV}$ and the sample thickness was $120 \mu \mathrm{m}$.

Overview of our experimental techniques were shown in Ref.13.

\section{Results and Discussion}

The transmission and SAXS measurements of Se70-Te30 mixtures were carried out from 400 to $1000{ }^{\circ} \mathrm{C}$ at 6 and $100 \mathrm{MPa}$. Temperature dependence of the density estimated by the transmission is shown in Fig.2(a). In our method, observed total transmission is affected by not only by the sample density but also by He gas density and the latter contribution is very large $\mathrm{e}^{12}$. Thus the absolute value of the sample density is less accurate and the estimated value is arbitrary one at the present stage. With increasing temperature, the density first normally decreases but anomalously increases from $650{ }^{\circ} \mathrm{C}$. When the pressure is elevated, the data becomes rather scattered but similar result was obtained.

$Q$-dependence of the scattered x-ray intensity, $I(Q)$, at some temperatures and $6 \mathrm{MPa}$ are plotted in Fig.3. In the larger- $Q$ region $\left(\mathrm{Q}>3.5 \mathrm{~nm}^{-1}\right), I(Q)$ increases with $Q$ towards the first peak $\left(Q \sim 20 \mathrm{~nm}^{-1}\right)$ of the structure factor. In the smaller- $Q$ region $(\mathrm{Q}<3.5 \mathrm{~nm}-1)$, at $400{ }^{\circ} \mathrm{C}, I(Q)$ hardly changes with $Q$, but with increasing temperature, $I(Q)$ becomes larger and exhibits larger $Q$-dependence. To make the temperature dependence clearer, we fitted $I(Q)$ in the small-Q region $\left(1.0<Q<3.5 \mathrm{~nm}^{-1}\right)$ with a linear function,
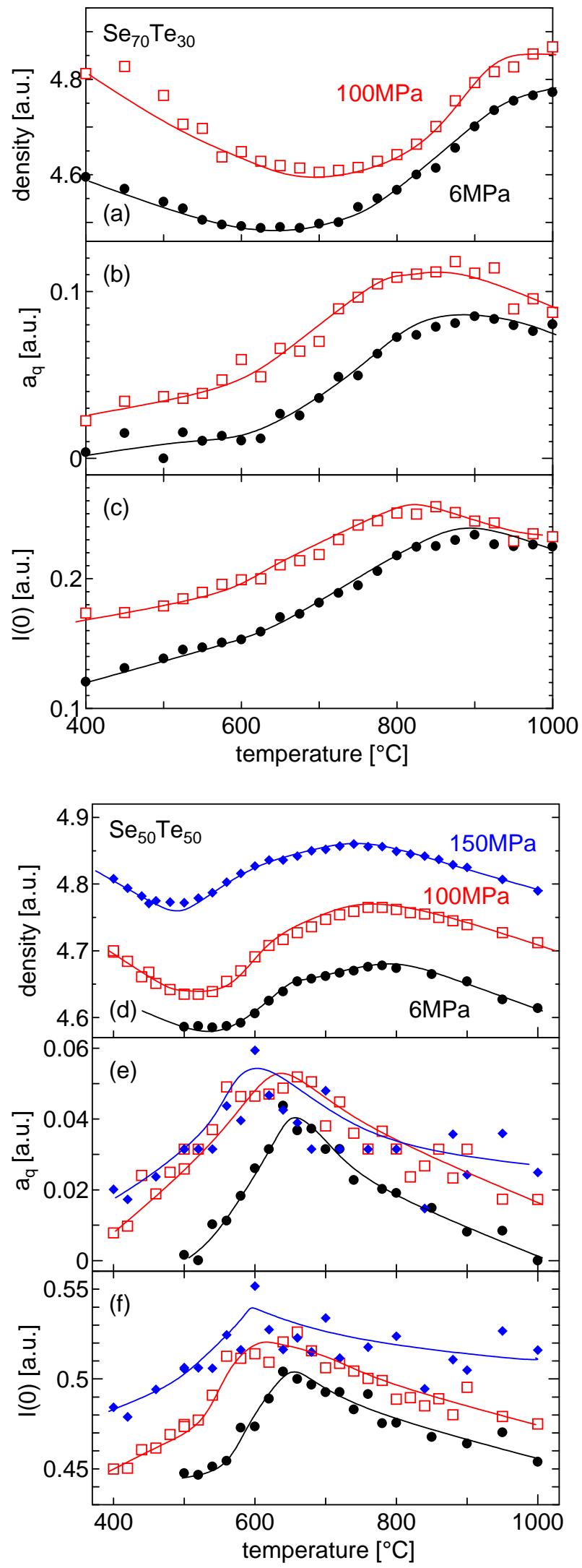

Fig. 2. Temperature dependence of density and SAXS parameters $a_{q}$ and $I(0)$ estimated by the present experiment (Se70-Te30) (a-c) and the previous one (Se50-Te50) (d-f). 


$$
I(Q)=I(0)\left(1-a_{q} Q\right)(1.1)
$$

and obtained the fitting parameters, $I(0)$ and $a_{q}$. The results are plotted in Fig. 2 (b) and (c). With increasing temperature at $6 \mathrm{MPa}$, both $\mathrm{I}(0)$ and $a_{q}$ gradually increase and show broad maximum at around $900{ }^{\circ} \mathrm{C}$. When the pressure is elevated, the curve shifts to lower temperature side.

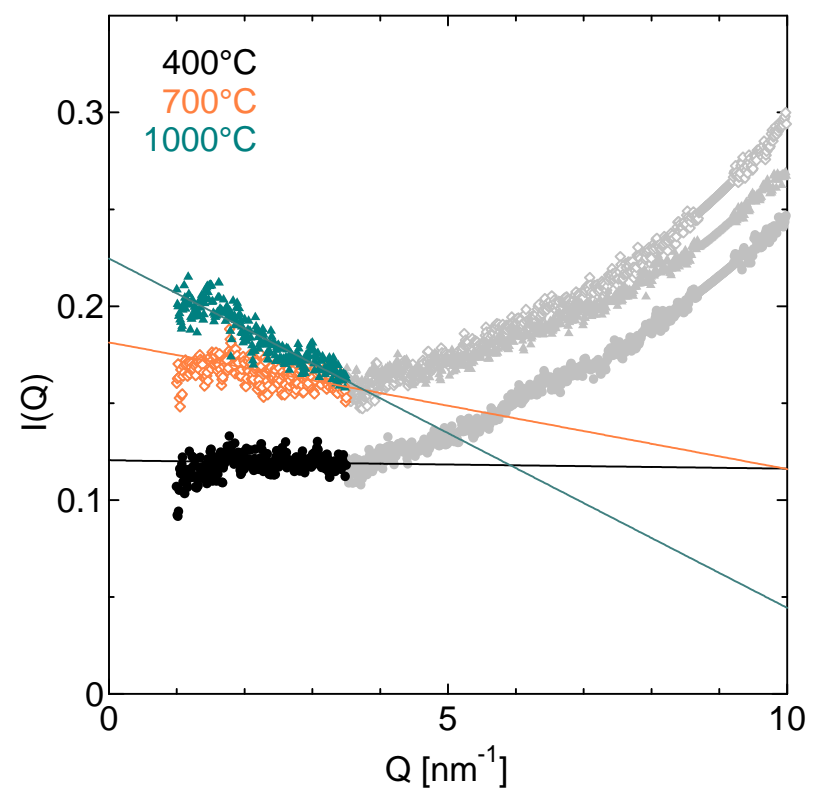

Fig. 3. Q-dependence of the scattering intensity. Solid lines are linear fitting results in the small-Q region (coloured region).

These results of Se70-Te30 are well explained by comparing with the previous results of Se50-Te50 mixtures ${ }^{12}$ as shown in Fig.2 (d-f). In Se50-Te50 at 100 $\mathrm{MPa}$, temperature dependence of the density shows minimum at around $500{ }^{\circ} \mathrm{C}$ and maximum at around 760 ${ }^{\circ} \mathrm{C}$. This behaviour can be interpreted that there are two (meta-)stable phases, low-density (below $500{ }^{\circ} \mathrm{C}$ ) and high-density ones (above $760{ }^{\circ} \mathrm{C}$ ) that are similar to liquid Se and liquid Te, respectively, and in the intermediate temperature region, the sample shows continuous transition between these two phases. As a proof of that, $I(0)$ and $a_{q}$ increase and show maximum in the transition region. The density, $I(0)$ and $a_{q}$ curves of Se70-Te30 are just shifted to higher temperature side compared to those of Se50-Te50, which is consistent with the shift of the M-NM transition as shown in Fig.1.

We are now carefully analysing IXS data of Se50-Te50 mixture and we report the results only shortly. We analysed the data by Damped Harmonic Oscillator model to ascertain the longitudinal acoustic mode. The mode disperses much faster than the ultrasonic sound velocity ${ }^{5}$ (so-called "fast sound" state) and the temperature dependence of the velocity estimated by this dispersion curve is much different from that of the sound velocity. But the ratio of two velocities, strength of the "fast sound" seems to increase with approaching to transition region, which is similar to the behaviour of SAXS intensity. This is a supporting result of our suggestion that the "positiveness" of the dispersion curve $\mathrm{e}^{14}$ or the strength of "fast sound" is a good sign of dynamical inhomogeneity ${ }^{14}$.

\section{Summary}

We have carried out density, SAXS and IXS measurements of liquid Se-Te mixtures. The temperature dependences of the density probe that there is a gradual phase transition from low-density phase to high-density one. In the transition region, SAXS intensities increase which indicates that the density inhomogeneity becomes larger. In the transition region, the strength of "fast sound" also increases and we can speculate that it is a good sign of dynamical inhomogeneity.

\section{References}

1. W. Hoyer, E. Thomas and M. Wobst, Phys. Lett 30A, (1975) 1633

2. R. Bellissent and G. Tourand, J. Non-Cryst. Sol. 35, (1980) 1221

3. H. Thurn and J. Ruska, J. Non-Cryst. Sol. 22, (1976) 331

4. M. Yao, K. Suzuki and K. Endo, Sol. State Comm. 34, (1980) 187

5. Y. Tsuchiya and E. F. W. Seymour, J. Phys. C 15, (1982) L687

6. S. Takeda, H. Okazaki and S. Tamaki, J. Phys. Soc. Jpn. 54 (1985) 1890

7. J. C. Perron, Adv. Phys. 16 (1967) 657

8. J. A. Gardner and M. Cutler, Phys. Rev. B 20, (1979) 529

9. M. Yao, M. Misonou, K. Tamura, K. Ishida, K. Tsuji and H. Endo, J. Phys. Soc. Jpn. 48 (1980) 109

10. M. Misonou and H. Endo, J. Phys. Soc. Jpn. 51, (1982) 2285

11. T. Takimoto and H. Endo, Phys. Chem. Liq. 12, (1982) 141

12. Y. Kajihara, M. Inui, K.Matsuda and Y. Tomioka, J. Phys.: Conf. Ser. 215, (2010) 012078

13. K. Tamura and M. Inui, J. Phys.: Condens. Matter 13, (2001) R337

14. Y. Kajihara, M. Inui, S. Hosokawa, K. Matsuda and A Q R Baron, J.Phys. : Condens. Matter 20 (2008) 494244 